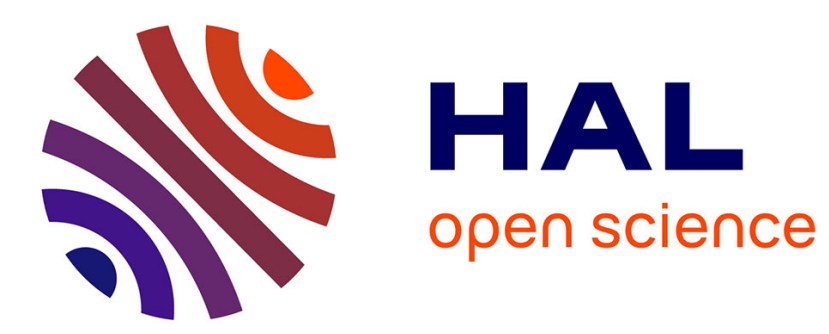

\title{
Comparative study of nonlinear crystals for electro-optic Q-switching of laser resonators
}

\author{
Jean-Paul Salvestrini, Mustapha Abarkan, Marc Fontana
}

\section{To cite this version:}

Jean-Paul Salvestrini, Mustapha Abarkan, Marc Fontana. Comparative study of nonlinear crystals for electro-optic Q-switching of laser resonators. Optical Materials, 2004, 26, pp.449-458. 10.1016/j.optmat.2003.10.009 . hal-00186026

\section{HAL Id: hal-00186026 \\ https://hal.science/hal-00186026}

Submitted on 2 Dec 2021

HAL is a multi-disciplinary open access archive for the deposit and dissemination of scientific research documents, whether they are published or not. The documents may come from teaching and research institutions in France or abroad, or from public or private research centers.
L'archive ouverte pluridisciplinaire HAL, est destinée au dépôt et à la diffusion de documents scientifiques de niveau recherche, publiés ou non, émanant des établissements d'enseignement et de recherche français ou étrangers, des laboratoires publics ou privés.

\section{다)(1) $(5$}

Distributed under a Creative Commons Attribution - NonCommerciall 4.0 International 


\title{
Comparative study of nonlinear optical crystals for electro-optic Q-switching of laser resonators
}

\author{
J.P. Salvestrini, M. Abarkan, M.D. Fontana \\ Laboratoire Matériaux Optiques, Photonique et Systèmes, FRE CNRS 2304, Université de Metz et Supélec, 2, rue E. Belin, 57070 Metz, France
}

The development of Pockels cells is closely related to the choice of the crystals which are involved in this kind of device. These crystals must satisfy several requirements. In order to clearly establish the crystal ability for the realization of Pockels cells, we define several figures of merit describing its performances for electro-optic Q-Switch and we use these figures of merit to compare the efficiencies of several NLO crystals.

\section{Introduction}

Laser activity can start when the threshold condition is met. The optical amplification for one round trip in the laser resonator is greater than the losses (due to output coupling, diffraction, absorption, scattering). The laser continues emitting until either the stored energy is exhausted, or the input from the pump source stops. Only a fraction of the storage capacity is effectively used in the operating mode. If it is possible to block the laser action long enough to store a maximum energy, then this energy could be released in a very short time period.

A method to achieve pulse laser emission is provided by Q-switching. The resonator quality, which represents a measure of the losses in the resonator, is kept low until the maximum energy is stored. A rapid increase of the resonator quality then takes the laser high above threshold, and the stored energy can be released in a very short time. The resonator quality can be controlled as a function of time in a number of ways. In particular, deep modulation of the resonator quality is possible with components that influence the state of polarization of the light. Rotating the polarization plane of linearly

Corresponding author.

E-mail address: salvestr@metz.supelec.fr (J.P. Salvestrini). polarized light through $90^{\circ}$, the light can be guided out of the laser at a polarizer.

For this, it is now well established that the linear (Pockels) EO effect is one of the most convenient properties that could be exploited to obtain fast Qswitching of laser resonator. Over the past few years, many studies have been undertaken in the use of nonlinear optical crystals which could improve the performances of Pockels cells (PC). These studies bring out the advantages and drawbacks of the different crystals analyzed.

Initially, water soluble crystals of potassium dihydrogen phosphate (KDP) and its isomorphs [1] were widely used in the longitudinal mode, i.e. when an electric field was applied along the direction of the optical beam. The KDP-family crystals are hygroscopic and require hermetic housing with protective windows, but have high optical uniformity and are useful for large aperture applications.

$\mathrm{LiNbO}_{3}$ (LN) offers good transmission and high extinction ratio with modest driving voltage in transverse configuration, i.e. when an electric field is applied perpendicular to the direction of the optical beam and it is not hygroscopic. When it is grown in its usual composition, i.e. congruent composition without any doping, it suffers from a relatively low optical damage threshold which is a major drawback. On the contrary, 
using LN crystals with stoichiometric composition seems to be a way to increase the damage threshold.

Another way to accomplish higher damage threshold could be the use of $\mathrm{LiTaO}_{3}$ (LT) crystals which are isomorphic to $\mathrm{LN}$ and also possess large $\mathrm{EO}$ properties, but with a damage threshold twice as large as in LN. It is to be noticed that both $\mathrm{LN}$ and LT exhibit large piezo-electric ringing when the electric field is applied along the dielectric $x$ - or $y$-axes. The effect of this piezoelectric ringing on the laser output may be the generation of multiple Q-switched pulses.

High damage threshold $\beta-\mathrm{BaB}_{2} \mathrm{O}_{4}$ (BBO) crystals have been employed lately in small aperture $\mathrm{PC}$, despite their low EO coefficients and short dimensions [2] (yielding large half-wave voltage $\left(V_{\pi}\right)$ ). The BBO Qswitches have been reported to exhibit negligible piezoelectric ringing [3].

Good Q-switching properties have been demonstrated with $\mathrm{KTiOPO}_{4}$ (KTP) crystals when operated in a thermally compensated mode. It also has large linear EO coefficients without piezoelectric ringing and high optical damage thresholds [4].

In addition to these crystals, during the last few years, newly developed nonlinear optical (NLO) crystals $\mathrm{GdCa}_{4} \mathrm{O}\left(\mathrm{BO}_{3}\right)_{3}(\mathrm{GdCOB})$ and $\mathrm{YCa}_{4} \mathrm{O}\left(\mathrm{BO}_{3}\right)_{3}$ (YCOB) have attracted much attention due to their optical properties [6-8]. They possess many advantages such as nonhygroscopy, wide transmission bands and good mechanical properties. Most of the research involving GdCOB and YCOB has been focused on the aspects of second-harmonic generation and self-frequency doubling. Although they have low electro-optic coefficient values, GdCOB and YCOB might be excellent candidates for EO Q-switching of high power and short wavelength laser resonators.
In the present work, to clearly establish the crystal's ability to realize PC, we will first define several figures of merit describing the performances needed for the EO Qswitch process. Then we will determine the values of the different coefficients involved in these figures of merit and finally we will compare the EO Q-switching efficiencies of the nonlinear crystals mentioned above.

\section{The different EO Q-switching operating modes}

Here, we recall the three operating modes that can be used for the EO Q-switching of a laser resonator. These three operating modes are called quarter-wave voltage on Q-switching operation, quarter-wave voltage off Qswitching operation and half-wave voltage operation, respectively.

The two quarter-wave operation modes are illustrated in Fig. 1. The off Q-switching operation (Fig. 1a) needs a PC and a single polarizer. It is the most economical mode with regards to the components used, and the simplest arrangement for Q-switching with an EO crystal. Light emitted by the laser rod is linearly polarized by the polarizer. If a quarter-wave voltage is applied to the PC, then on exit, the light is circularly polarized. After reflection from the resonator mirror and a further passage through the $\mathrm{PC}$, the light is once again polarized, but the plane of polarization has rotated $90^{\circ}$. The light is deflected out of the resonator at the polarizer, but the resonator quality is low and the laser does not oscillate. At the moment the maximum storage capacity of the active medium has been reached, the voltage of the PC is turned off very rapidly; the resonator quality increases immediately and a very short laser pulse is emitted.

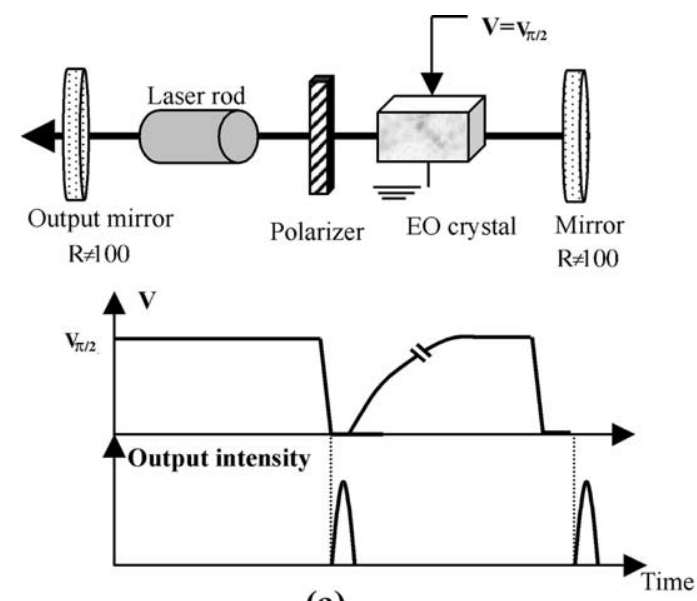

(a)
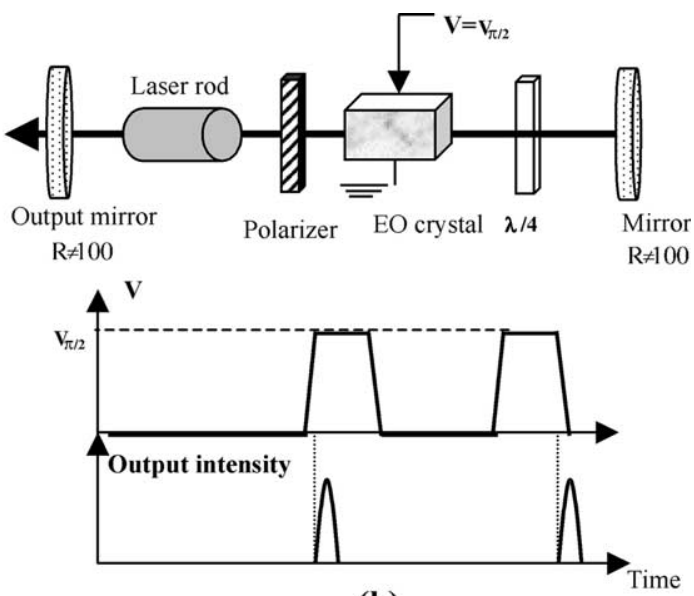

(b)

Fig. 1. Quarter-wave operating modes. (a) Off Q-switching, the quarter-wave voltage is applied to prevent lasing and is switched down to zero to generate the output laser pulse. (b) On Q-switching, quarter-wave voltage is required only during the time necessary to generate the output pulse laser. 
In addition to the PC and the polarizer, the second Qswitching operating mode (Fig. 1b) requires a quarterwave plate between the PC and the resonator mirror. If no voltage is applied to the $\mathrm{PC}$, the laser resonator is blocked: no laser action takes place. A voltage pulse opens the resonator causing the emission of laser light.

The selection of the proper Q-switch for a particular application results from the kind of laser stimulation, the desired pulse parameters, the switching voltage and switching speed of the PC. Basically, both off and on Qswitches are equivalent in physical terms for both $\mathrm{cw}$ and pulse pumped lasers. On Q-switching is, however, recommended in $\mathrm{cw}$ operation because a high voltage pulse and not a rapid switch-off is necessary to generate a laser pulse. This method also extends the life-time of the PC. Over a long period of time, the continuous application of a high voltage would lead to electrochemical degradation effects. Off Q-switching is more advantageous for laser stimulated with flash lamps because the quarter-wave plate is not required.

The third operating mode, half-wave operation, requires a PC and two polarizers. The disadvantage is that the half-wave voltage must be applied as a DC level which is switched off to generate the laser pulse. The major advantage of using two polarizers is evident in high power resonators where the second polarizer provides improved holdoff of conventional lasing.

\section{Figures of merit of electro-optic Pockels cells}

\subsection{Growth and preparation of the crystals}

A PC requires bulk materials $\left(\mathrm{a}\right.$ few $\mathrm{cm}^{3}$ ) contrary to the integrated systems which are presented in waveguides or thin films. The growth of materials used for this application must thus be able to provide large volumes of crystals. One of the major difficulties of bulk crystal growth is conserving the homogeneity and purity in the entire volume.

Once grown, the crystals will be cut and polished. This requires that the material be chemically stable (i.e. inert compared to traditional solvents: acetone, alcohol, distilled water) and sufficiently hard to allow high quality polishing.

The materials must also have phase transformation temperatures (phase transition, melting...) far from the temperatures related to the various technological preparation steps.

\subsection{Driving voltage and power}

One of the most significant characteristics of the EO $\mathrm{PC}$ is the voltage which has to be applied. As mentioned above, a PC alters the polarization of the transmitted light beam when the voltage is applied to the cell by causing phase retardation between orthogonal polarization components of the beam.

In the absence of an applied field, there is no difference in phase retardation between orthogonal polarization components of the light beam because the refractive index is the same for both polarization directions and so there is no polarization change in the light transmitted. However, an applied electric field creates fast and slow axes at $90^{\circ}$ to one another. The difference in velocity for beams with polarization components along these two directions, when voltage is applied, retards the phase of one polarization component relative to the other thereby changing the polarization state of the emerging beam.

The relative phase retardation, $\Gamma$, is given by the following expression:

$\Gamma=\frac{2 \pi L}{\lambda} \Delta n$

Here, $\Delta n$ is the birefringence (the difference in refractive index for the two polarizations of light), $L$ is the crystal length along the direction of the propagating beam, and $\lambda$ is the wavelength of light.

For the special case when the retardation $\Gamma$ is $\pi / 2$, or the quarter-wave value, the transmitted light is circularly polarized; when the retardation $\Gamma$ is $\pi$, which is the halfwave retardation value, the polarization of the transmitted light rotates $90^{\circ}$.

The expression that relates $\Delta n$ to the electric field is generally of the form below:

$\Delta n=\frac{n_{\mathrm{eff}}^{3} r_{\mathrm{eff}} E}{2}$

where $r_{\text {eff }}$ is the effective EO coefficient, $E$ is the electric field that is experienced by the crystal with the application of an applied voltage $V$, and $n_{\text {eff }}$ is the effective refractive index.

Taking into account Eqs. (1) and (2), we obtain the half-wave voltage as

$V_{\pi}=\frac{\lambda d}{n_{\mathrm{eff}}^{3} r_{\mathrm{eff}} L}$

where $d$ is the crystal thickness along the direction of the applied electric field.

This relation shows that the ratio $L / d$ could be adjusted, in the case of a transverse configuration (where the propagation direction of the light beam is perpendicular to the direction of the applied electric field) to obtain a small driving voltage. This is not possible in the longitudinal configuration (for which the light propagation direction and the applied electric field direction are collinear) since in this case the ratio $L / d$ is equal to 1 .

We can thus define a first figure of merit related to the driving voltage. This definition depends on the operating mode of the device. In the case of the on Q-switch mode it is the EO response at short time, characterized by the constant strain (clamped) EO coefficient $r_{\text {eff }}^{\mathrm{S}}$, which is 
important since the voltage is applied as short pulses. On the contrary, in the off Q-switch operating mode, the applied voltage is a DC voltage and thus it is the EO response at long time, characterized by the constant stress (unclamped) EO coefficient $r_{\text {eff }}^{T}$, which is preponderant. The definition of the related figure of merit $F_{1}$ is then twice:

$F_{1}=\frac{n_{\mathrm{eff}}^{3} r_{\mathrm{eff}}^{T \text { or } S} L}{d}$

This figure $F_{1}$ should be as large as possible to indicate that it is necessary to use materials having high values of refractive indices and EO coefficients. The dimension ratio $L / d$ is a parameter that should be as large as possible to get the smallest driving voltage.

In the on Q-switch operating mode, the voltage is applied as a short pulse. Thus the average driving power can be expressed as

$P_{\text {avg }}=\frac{V_{\pi}^{2} C f_{\text {rep }}}{2}$

where $f_{\text {rep }}$ is the repetition frequency of the applied voltage pulses and $C$ is the electrical capacity of the sample. Substituting Eq. (3) in Eq. (5) and using the relation between the capacity and the dielectric permittivity, the average driving power becomes:

$P_{\text {avg }}=\frac{2 \lambda^{3} \epsilon d^{2}}{\pi n_{\text {eff }}^{7} r_{\text {eff }}^{2} L^{2}} f_{\text {rep }}$

The EO coefficient as well as the dielectric permittivity constant $\epsilon$ which are to be taken into account in Eq. (6) are again the clamped quantities. We can thus define a second figure of merit related to the average driving power:

$F_{2}=\frac{n_{\mathrm{eff}}^{7}\left(r^{\mathrm{S}}\right)^{2} L^{2}}{\epsilon^{\mathrm{S}} d^{2}}$

Likewise as the figure of merit $F_{1}$, the ratio of the dimensions $L / d$, the values of the refractive index and of the EO coefficient have to be as large as possible. The other parameter is the dielectric permittivity constant which should have the smallest value possible.

\subsection{Pockels cell switching speed}

The output laser pulse characteristics (duration and peak power) are closely related to the speed of the Qswitching. The switching time $t_{r}$ depends strongly on the impedance of the load which is connected to the PC electronic driver. This impedance depends on the EO crystal which is essentially capacitive and on the resistivity $R_{\text {eq }}$ of the electronic circuitry. The switching time $t_{r}$ can be thus expressed as

$t_{r}=R_{\mathrm{eq}} \epsilon L$
Usually the crystals used in PC are prepared with a square section so that the dimensions $l$ and $d$ are equal. We can then introduce the following figure of merit:

$F_{3}=\frac{1}{L \epsilon^{\mathrm{S}}}$

$F_{3}$ requires as with $F_{2}$ that the dielectric permittivity constant have the smallest value possible. However, the crystal length should be small to minimize the switching time of the PC, contrary to the resulting figures of merit $F_{1}$ and $F_{2}$.

\subsection{Thermal effects}

A crystal possesses different properties according to its structure including the temperature. A variation of temperature in a PC can induce temporal drifts in its operation, but can also lead to a complete dysfunction (change of symmetry for instance), or even to the destruction of the crystal.

In a laser resonator, the heating of the $\mathrm{PC}$ can be consequential. Indeed, the crystal can undergo a large increase of temperature due not only to variations in ambient temperature but also to the absorption (even weak) of part of the luminous energy circulating in the resonator.

It is important to define a figure of merit related to absorption $\alpha_{\mathrm{ab}}$ and thus to the problem of heating by

$F_{4}=\frac{1}{\alpha_{\mathrm{ab}} L}$

Figure $F_{4}$ must be as high as possible so that the material possesses a low absorption coefficient and a small length in the propagation direction of the light beam. The refractive indices, as an intrinsic property of material, depend on the temperature as well. Consequently, because of the thermo-optical effect, any variation of temperature induces a phase shift given by

$\Gamma=\frac{2 \pi L}{\lambda} \frac{\delta \Delta n_{\mathrm{eff}}(T)}{\delta T} \Delta T$

This phase shift $\Gamma$ modifies the polarization state of the light which passes through the crystal and thus influences PC operation. A temperature variation induced by the absorption of the light can thus lead to the undesired opening of the laser resonator. Another effect to be considered concerns the thermal gradients that can appear in the crystal causing a spatial variation of the refractive index yielding a deflection of the light beam (thermal lens). This gradient of the refractive index is proportional to the thermo-optic coefficient $\frac{\partial n_{\text {eff }}}{\partial T}$ and to the inverse of the thermal conductivity $K$ as

$\Delta n_{\text {eff }}(z) \propto-\frac{1}{K} \frac{\partial n_{\text {eff }}}{\partial T} z^{2}$

where $z$ is the spatial coordinate along the propagation direction of the light beam. 


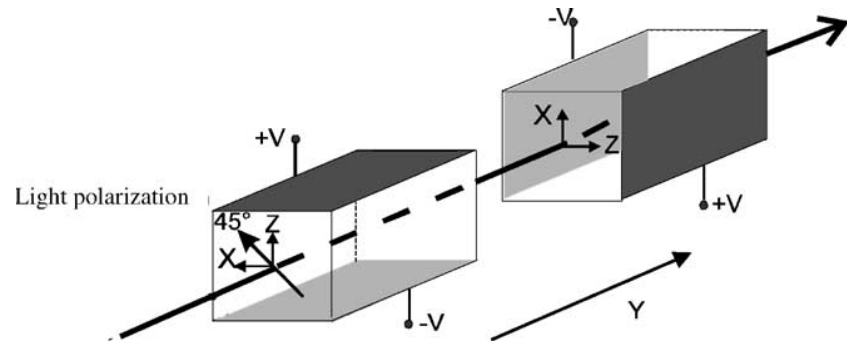

Fig. 2. Description of the sample mounting to compensate the thermooptic effect. $X, Y$ and $Z$ are the dielectric axes.

It is thus appropriate to introduce a new figure of merit characterizing this effect as

$F_{5}=\frac{K}{L \frac{\partial n_{\mathrm{eff}}}{\partial T}}$

The crystals which could be used in PC must have high thermal conductivity as well as refractive indices and birefringence nearly independent of temperature. The nonsensitivity of birefringence to temperature is obtained when a light beam propagates along an isotropic axis (optical axis). If this geometry is unavailable or inefficient, the use of two identical crystals in a compensating mode [2] can be an alternative, as shown on Fig. 2.

\subsection{Piezoelectric ringing}

The EO effect, which is at the origin of the Qswitching of the laser resonator, generally includes an acoustic contribution [9] linked to the piezo-electric deformation induced by the applied electric field. This effect appears in the devices as piezoelectric ringing and can cause serious problems such as unwanted output laser pulses, the destruction of the electrodes, or that of the crystal. The acoustic contribution $r_{i j, k}^{\mathrm{a}}$ to the EO coefficient is given by [10]:

$r_{i j, k}^{\mathrm{a}}=\sum_{m, n} p_{i j, m n} d_{k, m n}$

where $p_{i j, m n}$ and $d_{k, m n}$ are the components of the photoelastic and piezo-electric tensors, respectively. If this acoustic contribution to the EO coefficient is large, the amplitude of the piezo-electric ringing can become significant. We can thus define a new figure of merit characterizing the sensitivity of the device to piezoelectricity by

$F_{6}=\left(r_{i j, k}^{\mathrm{a}}\right)^{-1}$

It is important to choose a material which presents a small acoustic contribution in the used configuration, and thus the largest possible figure of merit $F_{6}$. Nevertheless, the effect can be neutralized by appropriate timing of the electrical pulse to the Q-switch.

\subsection{Summary}

The figures of merit defined in the above paragraphs are summarized in Table 1. One can see that the crystal to be chosen must have weak absorptions and good mechanical quality. It is also to be noted that it must have the largest possible EO coefficients, refractive indices and thermal conductivity. The material or at least its EO coefficients and its natural birefringence have to be insensitive to the temperature. In addition, a dielectric permittivity constant and the lowest possible electric conductivity are required. Indeed, applying DC voltage to a conductive crystal (due to ion migration for instance) for long periods of time may result in permanent damage. The polished surface can become fogged and etched. All crystal surfaces, including those under the conductive electrodes can be similarly affected. It is obvious that a compromise between these various criteria is necessary and depends on the application which is chosen.

Therefore it would be more useful to define, in practice, only one criterion. This is why we can define a general figure of merit, noted $M$, which includes the whole set of figures of merit given by Table 1:

$M=\frac{n_{\text {eff }}^{10}\left(r_{\text {eff }}^{\mathrm{S}}\right)^{2} r_{\text {eff }}^{T} K}{\left(\epsilon_{r}^{\mathrm{S}}\right)^{2} r^{\mathrm{a}} L d}$

This new figure of merit will enable us to compare the different crystals which can be used to achieve a PC. In this way, any material under study for its possible insertion in a PC requires the following values to be known:

- EO coefficients and their frequency, wavelength and temperature dependence,

- refractive indices, their wavelengths and temperature (thermo-optic coefficient) dispersions,

- dielectric permittivity constants, their frequency and temperature dependence,

- thermal and electrical conductivity constants,

- absorption coefficients.

These various studies are to be complemented by the measurement and analysis of the piezoelectric ringing.

Table 1

Summary of the figure of merit characterizing a Pockels cell

\begin{tabular}{lll}
\hline Criterion & Figure of mrite & Definition \\
\hline Driving voltage & $F_{1}(\mathrm{pm} / \mathrm{V})$ & $n_{\mathrm{eff}}^{3} r_{\mathrm{eff}}^{T} \frac{L}{d}$ \\
Driving power & $F_{2}\left((\mathrm{pm} / \mathrm{V})^{2}\right)$ & $\frac{n_{\mathrm{eff}}^{7}\left(r_{\mathrm{ef}}^{\mathrm{S}}\right)^{2} L^{2}}{\epsilon_{r}^{\mathrm{f}} d^{2}}$ \\
Switching speed & $F_{3}\left(\mathrm{~cm}^{-1}\right)$ & $\frac{\frac{1}{\epsilon_{r}^{S} L}}{\epsilon^{S}}$ \\
Absorption & $F_{4}\left(\mathrm{~cm}^{-1}\right)$ & $\frac{1}{a_{\mathrm{abb}} L}$ \\
Thermal effect & $F_{5}\left(\mathrm{~mW} \mathrm{~cm}^{-2}\right)$ & $L \frac{k}{\partial e_{\mathrm{eff}}}$ \\
Piezoelectric ringing & $F_{6}\left((\mathrm{pm} / \mathrm{V})^{-1}\right)$ & $\left(r_{i j, k}^{\mathrm{a}}\right)^{-1}$ \\
\hline
\end{tabular}




\section{Characterizations}

\subsection{Electro-optical and thermo-optical characterizations}

The experimental set-up used to measure the EO coefficients was based on the Sénarmont arrangement (see Fig. 3).

The crystal followed by a fixed $\lambda / 4$ plate was located between a linear polarizer and an analyzer. The polarizer was set to yield the laser light polarized at $45^{\circ}$ to the principal axis of the crystal. The $\lambda / 4$ plate axis was also set at $45^{\circ}$ to the crystal axis, while the analyzer was oriented at an azimuthal angle $\beta$. The transfer function of the light intensity transmitted through the setup can be written as [11]:

$T=\frac{1-\gamma \sin (\Gamma-2 \beta)}{2}$

where $\beta$ is the angular position of the analyzer and $\Gamma$ is the phase shift introduced by the EO crystal, respectively, whereas

$\gamma=\frac{I_{\max }-I_{\min }}{I_{\max }+I_{\min }}$

is the contrast which is usually considered as a constant parameter. The phase shift $\Gamma_{E}$ and the birefringence $\Delta n(E)$ induced by electric field $E$ are given by

$\Gamma_{E}=\frac{2 \pi L}{\lambda} \Delta n(E)=\frac{\pi}{\lambda} L n_{\mathrm{eff}}^{3} r_{\mathrm{eff}} E$,

where $L$ is the length of the crystal along the beampropagation direction, $\lambda$ is the wavelength, $n_{\mathrm{eff}}$ and $r_{\mathrm{eff}}$ are the effective refractive index and EO coefficient, respectively.

We used the time response method (TRM) which is able to provide the frequency dispersion of the EO coefficients. In particular, we determined the clamped (or constant strain) $r^{\mathrm{S}}$ coefficient and the unclamped (or constant stress) $r^{T}$ coefficient. The method consisted in measuring the time response of the EO crystal to a voltage step. To do this, a large voltage (up to $1 \mathrm{kV}$ ) pulse was switched on the crystal with a short rising time (a few nanoseconds) in order to obtain the value of coefficient $r^{\mathrm{S}}$ and with a long duration (several $\mu_{\mathrm{S}}$ ) to determine the value of coefficient $r^{T}$.

The voltage-induced intensity variation is at its highest at the linear part of the transfer function. At this working point, the time dependence of the variation of the transmitted beam intensity $\Delta i(t)$ induced by the applied voltage $\Delta V(t)$ yields the time response of the EO crystal under test as

$\Delta i(t)=\frac{\pi n_{\text {eff }}^{3} L I_{0}}{2 \lambda d} r_{\text {eff }}(t) \otimes \Delta V(t)$

where $\otimes$ is the convolution operator, $I_{0}=I_{\max }-I_{\min }$ represents the total intensity shift of the transfer function and $r_{\text {eff }}(t)$ is the value of the EO coefficient. The frequency dispersion of the EO coefficients can be derived from the ratio of the quantities $\Delta i(v)$ and $\Delta V(v)$ which are obtained by the $Z$-transformation of the signals $\Delta i(t)$ and $\Delta V(t)$ which were measured. We obtained

$r_{\mathrm{eff}}(v)=\frac{2 \lambda d}{\pi n_{\mathrm{eff}}^{3} I_{0} L} \frac{\Delta i(v)}{\Delta V(v)}$

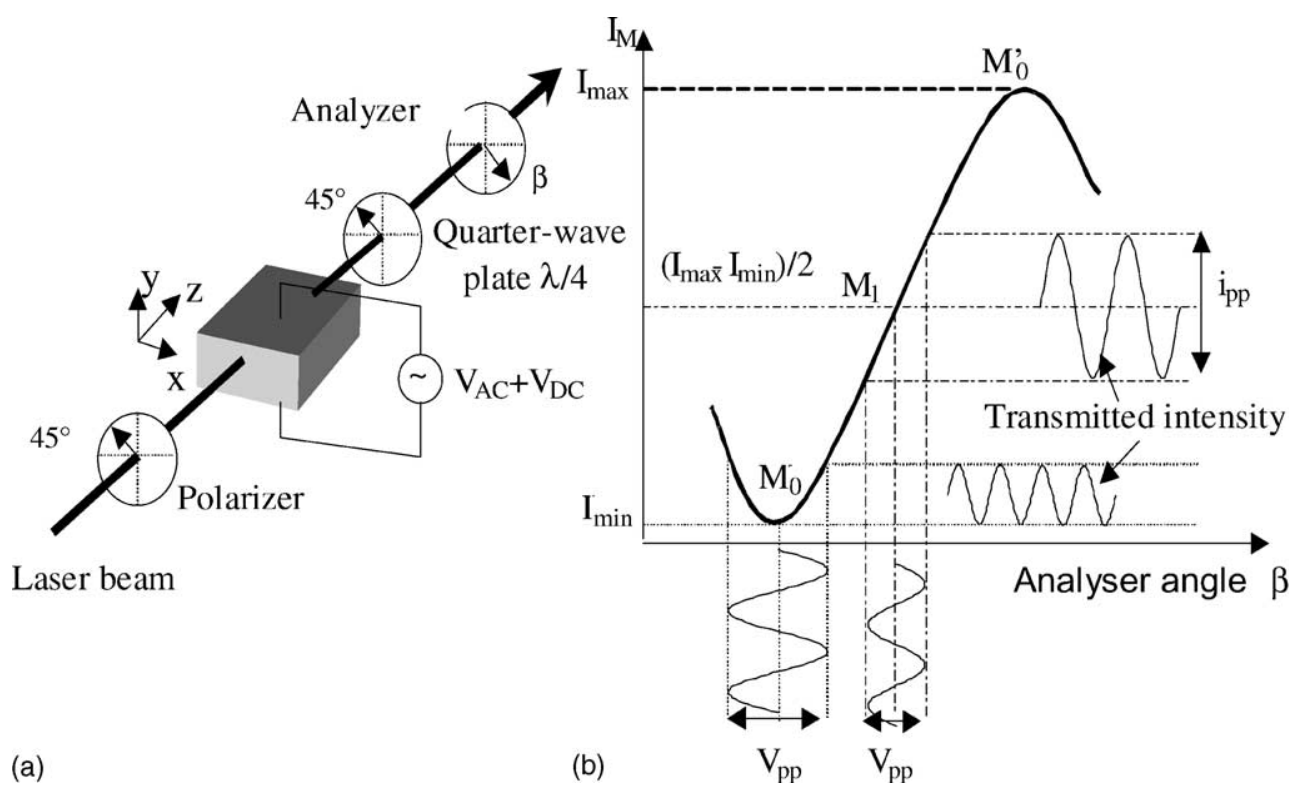

Fig. 3. (a) Sénarmont arrangement used for EO measurements. (b) Optical transmission versus the angle of analyzer $\beta$. Point $M_{0}$ is the minimum transmission point for which the output optical signal has a frequency that is twice as high as the frequency of the applied electric field. $M_{1}$ is the $50 \%$ transmission point yielding the linear replica of ac voltage. 





We have shown [11] that this technique allowed us to obtain the frequency dispersion of the EO coefficient from DC up to at least $150 \mathrm{MHz}$, depending only on the rising time of the voltage pulse.

It was used to characterize the following nonlinear optical crystals: commercial congruent LN crystals (LNc), stoichiometric LN crystals (LNs) prepared from high temperature top seeded solution growth techniques [5], BBO and YCOB crystals and commercial KTP and LT crystals. The different configurations used in our experiments are summarized in Table 2.

Fig. 4 shows examples of the dynamics observed in the EO response of samples under study according to Table 2. Experimental data are plotted on two timescales: on the shorter scale (Fig. 4a), the signal reaches a plateau immediately after the electric field has been switched on; this corresponds to the clamped EO response.

The acoustic phonon contributions became of importance only after several $100 \mathrm{~ns}$, and lead to clamped oscillations in the EO signal, as can be seen on a longer time-scale (Fig. 4b). The value of unclamped EO coefficient $r^{T}$ was obtained after a long time $(10 \mu \mathrm{s})$.

In Fig. 5, we present the frequency dependence of the EO coefficients deduced from the time response presented in Fig. 4 by using Eq. (21). The frequency range of our measurements in this case was equal to $150 \mathrm{MHz}$.

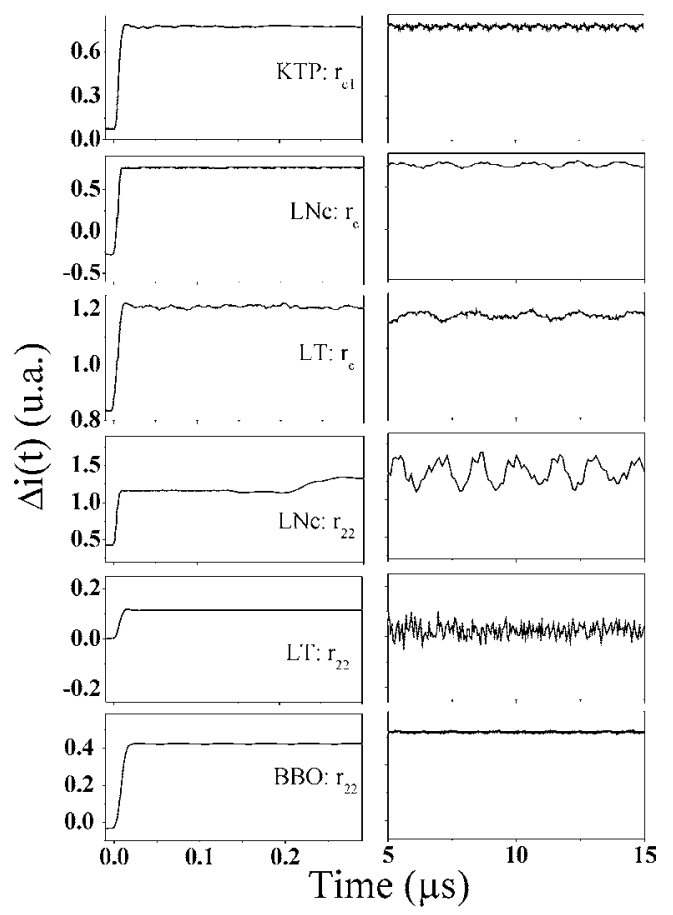

(a)

(b)

Fig. 4. Time resolved EO response to a step voltage. (a) In [0;0.3 $\mu \mathrm{s}]$ time-scale and (b) in $[5 ; 15 \mu \mathrm{s}]$ time-scale. Measurements were performed at the wavelength of $632.8 \mathrm{~nm}$.

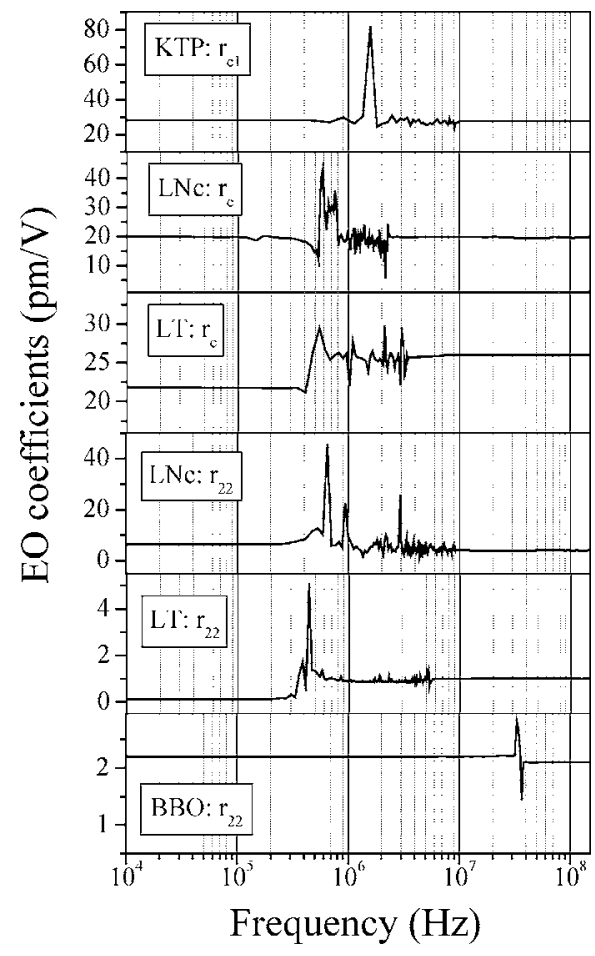

Fig. 5. Frequency dependence of the EO coefficients at a wavelength of $1064 \mathrm{~nm}$. The dependence was deduced from the results obtained during the EO time response measurements.

At higher frequencies the signal from the detection that was too noisy was not considered.

The values of the unclamped (constant stress) EO coefficients $r^{T}$ as deduced from data below the resonances are reported in Table 3. Above $10 \mathrm{MHz}$ and up to $150 \mathrm{MHz}$ the EO coefficients remained constant for each crystal with a value corresponding to the clamped (constant strain) EO coefficient (Table 3).

Furthermore, we derived the acoustic contributions to the EO effect from the difference between unclamped and clamped values $r^{\mathrm{a}}=r^{T}-r^{\mathrm{S}}$ (Table 3). Note that the plot of the frequency dependence of the EO coefficient $r_{\text {eff }}$ constitutes a direct method to determine $r^{\mathrm{a}}$, in contrast with the calculation of the acoustic contribution as the product of the components of the elasto-optic and piezo-electric tensors $p$ and $d$, respectively, according to Eq. (14). As we have shown above, the thermo-optic coefficient is required to calculate the figures of merit of the PC. Using the same measurement setup described in the previous section, it was possible to obtain the temperature dependence of the natural birefringence for each crystal and each configuration. To measure this thermo-optic coefficient, we used the working point $M_{0}$ (see Fig. 3b) at which the transmitted intensity was at its minimum (i.e. $I=I_{\min }$ ). An alternative electric field of frequency $v$ applied to the EO crystal generated an optical signal modulated at the double frequency $2 v$. The double-frequency signal was lost as a variation of 
Table 3

Unclamped $r^{T}$, clamped $r^{\mathrm{S}}$ EO coefficients and acoustic contribution $r_{\mathrm{a}}$ to the EO coefficients measured in congruent and stoichiometric LiNbO ${ }_{3}$, congruent $\mathrm{LiTaO}_{3}$, KTP, $\beta$-BBO and YCOB crystals at a wavelength of $1064 \mathrm{~nm}$

\begin{tabular}{llll}
\hline Crystal & Unclamped EO coefficient $r^{T}(\mathrm{pm} / \mathrm{V})$ & Clamped EO coefficient $r^{\mathrm{S}}(\mathrm{pm} / \mathrm{V})$ & Acoustic phonon contribution $r^{\mathrm{a}}(\mathrm{pm} / \mathrm{V})$ \\
\hline LNc & $r_{22}^{T}=6.4 \pm 0.3$ & $r_{22}^{\mathrm{S}}=3.8 \pm 0.2$ & $r_{22}^{\mathrm{a}}=2.6$ \\
& $r_{\mathrm{c}}^{T}=20 \pm 1$ & $r_{\mathrm{c}}^{\mathrm{S}}=20 \pm 1$ & $r_{\mathrm{c}}^{\mathrm{a}}=0$ \\
LNs & $r_{22}^{T}=6.8 \pm 0.4$ & $r_{22}^{\mathrm{s}}=4.5 \pm 0.2$ & $r_{22}^{\mathrm{a}}=2.3$ \\
& $r_{\mathrm{c}}^{T}=18 \pm 1$ & $r_{\mathrm{c}}^{\mathrm{S}}=18 \pm 1$ & $r_{\mathrm{c}}^{\mathrm{a}}=0$ \\
LT & $r_{22}^{T}=0.10 \pm 0.01$ & $r_{22}^{\mathrm{S}}=1.0 \pm 0.1$ & $r_{22}^{\mathrm{a}}=-0.90$ \\
& $r_{\mathrm{c}}^{T}=21.5 \pm 1.5$ & $r_{\mathrm{c}}^{\mathrm{S}}=26.5 \pm 1.5$ & $r_{\mathrm{c}}^{\mathrm{a}}=-5$ \\
$\mathrm{KTP}$ & $r_{\mathrm{c} 1}^{T}=28 \pm 2$ & $r_{\mathrm{c} 1}^{\mathrm{S}}=28 \pm 2$ & $r_{\mathrm{c} 1}^{\mathrm{a}}=0$ \\
$\mathrm{BBO}$ & $r_{22}^{T}=2.2 \pm 0.1$ & $r_{22}^{\mathrm{S}}=2.1 \pm 0.1$ & $r_{22}^{\mathrm{a}}=0.1$ \\
$\mathrm{YCOB}$ & $r_{\mathrm{c} 2}^{T}=1.65 \pm 0.1$ & $r_{\mathrm{c} 2}^{\mathrm{S}}=1.5 \pm 0.1$ & $r_{22}^{\mathrm{a}}=0.15 \pm 0.2$ \\
& $r_{a 1}^{T}=0.35 \pm 0.05$ & $r_{\mathrm{c} 2}^{\mathrm{S}}=0.25 \pm 0.05$ & $r_{22}^{\mathrm{a}}=0.1 \pm 0.1$ \\
\hline
\end{tabular}

Table 4

Values of dielectric permittivity (constant strain), thermo-optic coefficient (TO), electrical $(\sigma)$ and $(K)$ conductivity

\begin{tabular}{|c|c|c|c|c|c|c|c|}
\hline & $\overline{\epsilon_{x}^{\mathrm{S}}}$ & $\overline{\epsilon_{y}^{\mathrm{S}}}$ & $\overline{\epsilon_{z}^{\mathrm{S}}}$ & $\begin{array}{l}\text { TO cofficient } \\
\left(10^{-6} \mathrm{~K}^{-1}\right)\end{array}$ & $\begin{array}{l}\sigma \text { (along } z \text {-axis) } \\
\left(\Omega \mathrm{cm}^{-1}\right)\end{array}$ & $\mathrm{K}\left(\mathrm{mWcm}{ }^{-1} \mathrm{~K}^{-1}\right)$ & $n(1064 \mathrm{~nm})$ \\
\hline $\mathrm{LNc}$ & - & $56 \pm 3$ & $30 \pm 2$ & $\begin{array}{l}0^{(1)} \\
41 \pm 0.8^{(3)}\end{array}$ & $2.2 \times 10^{-17}$ & 56 & $\begin{array}{l}n_{0}=2.23 \\
n_{\mathrm{e}}=2.15\end{array}$ \\
\hline LNs & - & $45 \pm 2$ & $30 \pm 2$ & $\begin{array}{l}0^{(1)} \\
41 \pm 0.8^{(3)}\end{array}$ & - & & \\
\hline LT & - & $62 \pm 3$ & $41 \pm 2$ & $\begin{array}{l}0^{(1)} \\
49 \pm 1^{(3)}\end{array}$ & $4 \times 10^{-18}$ & - & $\begin{array}{l}n_{0}=2.13 \\
n_{\mathrm{e}}=2.04\end{array}$ \\
\hline KTP & - & - & $18 \pm 1$ & $31 \pm 0.5^{(4)}$ & $1.5 \times 10^{-7}$ & 33 & $n_{3}=1.86$ \\
\hline BBO & - & $9.4 \pm 0.5$ & & $0^{(1)}$ & $6.8 \times 10^{-18}$ & 12 & $n_{0}=1.64$ \\
\hline YCOB & $8.5 \pm 0.7$ & & $7.7 \pm 0.5$ & $\begin{array}{l}59 \pm 2^{(5)} \\
0^{(2)}\end{array}$ & $2 \times 10^{-13}$ & - & $n_{3}=1.7$ \\
\hline
\end{tabular}

The latter values were taken from the literature. ${ }^{(1)}$ : in configuration $r_{22},{ }^{(2)}$ : in configuration $r_{a 1},{ }^{(3)}$ : in configuration $r_{\mathrm{c}},{ }^{(4)}$ : in configuration $r_{\mathrm{cl}},{ }^{(5)}$ : in configuration $r_{\mathrm{c} 2}$.

temperature was introduced, and would be recovered if the analyzer was rotated by an angle $\beta$. The variation of the birefingence induced by the thermo-optic effect is given by [12]:

$\frac{\mathrm{d} \Delta n}{\mathrm{~d} T}=\frac{\lambda}{\pi L} \frac{\mathrm{d} \beta}{\mathrm{d} T}$

The results of these measurements carried out on all the crystals under study are reported in Table 4.

\subsection{Dielectric permittivity and electrical conductivity measurements}

Dielectric permittivity measurements were taken by means of a HP 4192 impedance analyzer using the same rhombic samples which were utilized during electrooptical and thermo-optical characterization. In Table 4, for each crystal tested, we report the value of the real part of the dielectric permittivity which was obtained for frequencies above piezoelectric resonances (clamped coefficient $\epsilon^{\mathrm{S}}$ ).

The principle that was used to determine the dark conductivity of the crystals was based on the thermal activation of the impurities present in the crystal. By extrapolation (via Arrhenius-type dependence) of the plot of the electrical current versus applied voltage at high temperature, it was possible to obtain the value of the dark conductivity.

These values are also reported in Table 4 along with the values of thermal conductivity and the refractive indices which were taken from the literature since they were needed to calculate the different figures of merit.

\section{Conclusion}

Finally, using all the measured values of the different properties, we calculated the values of the figures of merit for a wavelength of $1064 \mathrm{~nm}$ for all the crystals and for each configuration. The results are shown in Table 5.

We noticed that in the $r_{\mathrm{c}}$ configuration, the KTP crystals as well as the LN crystals in the two compositions exhibit the highest values for the figures of merit $F_{1}, F_{2}, F_{3}$, and $F_{6}$ compared to those of LT and YCOB crystals. This also appears in figure $M$. However, the KTP crystal has very high electrical conductivity 
Table 5

Values of the figures of merit for the different crystals

\begin{tabular}{|c|c|c|c|c|c|c|}
\hline Figure of merit & $F_{1}$ & $F_{2}$ & $F_{3}$ & $F_{5}$ & $F_{6}$ & $M$ \\
\hline \multicolumn{7}{|l|}{$\frac{\mathrm{d} \Delta n}{\mathrm{~d} T} \neq 0$} \\
\hline LNc, $r_{\mathrm{c}}$ & 181 & 2365 & 0.034 & $1.36 \times 10^{6}$ & 10 & $8 \times 10^{6}$ \\
\hline LNs, $r_{\mathrm{c}}$ & 173 & 2161 & 0.034 & $1.36 \times 10^{6}$ & 10 & $7 \times 10^{6}$ \\
\hline $\mathrm{LT}, r_{\mathrm{c}}$ & 170 & 1802 & 0.024 & $1.14 \times 10^{6}$ & 0.9 & $0.4 \times 10^{6}$ \\
\hline $\mathrm{KTP}, r_{\mathrm{cl}}$ & 171 & 2975 & 0.055 & $1.06 \times 10^{6}$ & 10 & $9 \times 10^{6}$ \\
\hline $\mathrm{YCOB}, r_{\mathrm{c} 2}$ & 8 & 12 & 0.13 & $0.2 \times 10^{6}$ & 6.7 & 1000 \\
\hline \multicolumn{7}{|l|}{$\frac{\mathrm{d} \Delta n}{\mathrm{~d} n}=0$} \\
\hline LNc, $r_{22}$ & 66 & 56 & 0.02 & - & 0.37 & 1532 \\
\hline LNs, $r_{22}$ & 69 & 92 & 0.022 & - & 0.43 & 3363 \\
\hline $\mathrm{LT}, r_{22}$ & 1 & 1.4 & 0.016 & - & 1.78 & 2 \\
\hline $\mathrm{BBO}, r_{22}$ & 9 & 12 & 0.1 & - & 10 & 1296 \\
\hline $\mathrm{YCOB}, r_{22}$ & 1.3 & 0.3 & 0.12 & - & 10 & 7 \\
\hline
\end{tabular}

In the calculation, we arbitrarily chose the crystal dimensions as $L=d=1$.

(compared to that of the other crystals) which leads to serious problems as we have seen above. Moreover, configuration $r_{\mathrm{c}}$ is very sensitive to low temperature variations and needs the use of two crystals to compensate the natural birefringence as was mentioned above. However, in configuration $r_{22}$ which is insensitive to temperature, all the crystals possess lower values of the figures of merit $F_{1}$ and $F_{2}$ than in configuration $r_{\mathrm{c}}$. Nevertheless, LN crystals, especially in stoichiometric compositions, exhibit higher values of the figures of merit compared to BBO and LT crystals. YCOB crystals have very low values of figures of merit even when compared to those of BBO crystals. Nevertheless, the possibility of obtaining a large-sized crystal (several centimeters), and thus a large value of the $L$ to $d$ ratio, along with the possibility of implementing several optical functions on the same substrate make YCOB crystals promising.

\section{Acknowledgements}

We are grateful to Katalin Polgár for supplying the lithium niobate and beta barium borate crystals, N. Téofanous for the lithium tantalate crystals, D. Pelenc for the YCOB crystals and D. Lupinski for the KTP crystals. This work was sponsored by the Region of Lorraine and the French Ministry of Research.

\section{References}

[1] W. Koechner, Solid State Laser Engineering, Springer-Verlag, Berlin, 1996.

[2] G.D. Goodno, Z. Guo, R.J.D. Miller, I.J. Miller, J.W. Montgomery, S.R. Adhav, R.S. Adhav, Appl. Phys. Lett. 66 (1995) 1575 .

[3] E. Lebiush, R. Lavi, Y. Tsuk, M. Winik, N. Angert, A. Gachechiladze, M. Tseitlin, A. Zharov, M. Roth, Ann. Israel Phys. Soc. 14 (2000).

[4] C.A. Ebbers, S.P. Velsko, Appl. Phys. Lett. 67 (1995) 593.

[5] W.D. Fountain, Appl. Opt. 10 (1971) 972.

[6] M. Iwai, T. Kobayashi, H. Furuya, Y. Mori, T. Sasaki, Jpn. J. Appl. Phys. 36 (1997) 276.

[7] W.K. Jang, Q. Ye, J. Eichenholtz, R. Peale, M. Richardson, A. Chin, B.H.T. Chai, Opt. Commun. 332 (1998) 155.

[8] Q. Ye, L. Shah, J. Eichenholtz, D. Hammons, R. Peale, M. Richardson, A. Chin, B.H.T. Chai, Opt. Commun. 33 (1999) 166.

[9] P. Amundsen, G. Wang, IEEE J. Quantum Electron. QE-23 (1987) 2252.

[10] M. Abarkan, J.P. Salvestrini, M.D. Fontana, M. Aillerie, Appl. Phys. B 76 (2003) 765.

[11] M. Abarkan, J.P. Salvestrini, M. Aillerie, M.D. Fontana, Appl. Opt. 42 (2003) 2346

[12] M. Aillerie, M.D. Fontana, F. Abdi, C. Carabatos-Nedelec, N. Thofanous, G.E. Alexakis, J. Appl. Phys. 65 (1989) 2406. 